\title{
A Consistent Construction of the Electromagnetic Energy-Momentum Tensor
}

\author{
Eliahu Comay \\ Charactell Ltd., Tel-Aviv, Israel \\ Email: elicomay@post.tau.ac.il
}

How to cite this paper: Comay, E. (2018) A Consistent Construction of the Electromagnetic Energy-Momentum Tensor. Open Access Library Journal, 5: e4354. https://doi.org/10.4236/oalib.1104354

Received: January 22, 2018

Accepted: February 8, 2018

Published: February 11, 2018

Copyright (๑) 2018 by author and Open Access Library Inc.

This work is licensed under the Creative Commons Attribution International License (CC BY 4.0).

http://creativecommons.org/licenses/by/4.0/

\begin{abstract}
This work discusses the problem of the apparently non-symmetric form of the electromagnetic fields' energy-momentum tensor, which is obtained from the variational principle. The analysis treats differently radiation fields and bound fields. This distinction has a solid experimental basis where the hydrogen atom proves that radiation fields and bound fields have a different spin and a different parity. A direct calculation proves that in the case of radiation fields, the variational principle yields the well known symmetric energy momentum tensor and the problem does not exist.
\end{abstract}

\section{Subject Areas}

Theoretical Physics

\section{Keywords}

Lagrangian Density, Energy-Momentum Tensor, Radiation Fields, Bound Fields

\section{Introduction}

The energy-momentum tensor of electromagnetic fields (called also stress tensor) represents the fields' energy-momentum density and current (see [1], pp. 86, 87; [2], pp. 601-605). As a tensor in Minkowski space, it is a part of the relativistic description of classical electrodynamics. This energy-momentum tensor is an indispensable element of this theory because it is used in a proof of local conservation of energy and momentum (see [1], pp. 88, 89; [2], pp. 606-607).

The standard procedure of constructing this tensor uses the invariance of the fields' Lagrangian density under a space-time translation. A general argument proves that this tensor conserves energy-momentum and that it should be symmetric (see [1], pp. 82-85). However, it turns out that in the case of 
electromagnetic fields, the tensor obtained from this procedure is not symmetric and a specific mathematical trick is used for symmetrizing it (see [1], pp. 86, 87; [2], pp. 604-605). This is certainly a problem of the theory because one expects that a fundamental argument which relies on the fields' Lagrangian density should directly yield a consistent expression for the energy-momentum tensor. The following words nicely describe this problem (see the first paragraph of [3]). "Few things are more frustrating to students than to be led through a long, formal argument only to be told at the end that the result obtained is incorrect and must somehow be fixed by an auxiliary procedure. This is particularly harmful if the formal argument involved turns out to be one of the mathematical cornerstones of modern physics. Unless the discussion includes a re-examination of the analysis to find out exactly what went wrong, the students will be left with the paradoxical feeling that a supposedly very general theorem produces unacceptable answers when applied to certain specific situations. Quite understandably, later on they will be reluctant to think about any physical problem in terms of the tools provided by such a theorem."

The situation described by this quotation indicates that the problem requires "a re-examination of the analysis to find out exactly what went wrong" in the standard construction of the energy-momentum tensor of electromagnetic fields. This issue is the main objective of the present work. The analysis refrains from using specific assumptions that directly aim to correct the problem. Instead, it relies on well established experimental evidence that affects the structure of the electromagnetic part of the Lagrangian density.

Units where $\hbar=c=1$ are used. Greek indices run from 0 to 3 and Latin indices run from 1 to 3 . The metric $g^{\mu v}$ is diagonal and its entries are $(1,-1$, $-1,-1)$. The symbol,$\mu$ denotes the partial differentiation with respect to $x^{\mu}$. The second section proves that radiation fields and bound fields are different physical objects. The third section shows how the well known symmetric tensor is directly obtained from the Lagrangian density of radiation fields. The fourth section shows how this tensor is obtained for bound fields. The fifth section contains a discussion of the results. The last section contains a summary of this work.

\section{Radiation Fields and Bound Fields}

Let us examine physical properties of electromagnetic fields (see Section 4 in [4]). For the completeness of the presentation, this analysis is briefly repeated here. The hydrogen atom is used as the experimental device. Since quantum mechanics provides an excellent description of properties of the hydrogen atom, quantum textbooks are used here as a reliable basis.

Maxwellian electrodynamics proves the existence of radiation fields (see [1], pp. 184-187; [2], pp. 391-397). Outgoing radiation fields emitted by a given system of charges are the fields of the system's charges at the wave zone (see [1], p. 184; [2], p. 392). (The wave zone is also called far zone or radiation zone.) For 
a given system of charges, incoming radiation fields are the outgoing radiation fields of other systems of charges.

Let us examine the interaction of the hydrogen atom with an incoming radiation. Here the atom can absorb the photon's energy if the energy of the final state equals the energy of the initial state plus the photon's energy and the angular momentum of the initial state $l$ and that of the final state $l^{\prime}$ satisfy

$$
l^{\prime}-l= \pm 1
$$

(see [5], p. 264; [6], p. 466). It follows that the photon, which is the quantum form of electromagnetic radiation, has spin-parity $j^{\pi}=1^{-}$. These facts are recognized by an authorized institute (see the data on the $\gamma$ photon here [7]).

On the other hand, let us examine the quantum description of the ground state of the hydrogen atom. Here the electron is in an s-wave and the electromagnetic binding energy is

$$
E_{b}=\int \psi^{\dagger} \frac{e^{2}}{r} \psi \mathrm{d}^{3} r
$$

Energy is the 0-component of the energy-momentum 4-vector. Therefore, the left hand side of (2) is a scalar in the 3-dimensional space. Let us evaluate the right hand side of (2). The angular momentum of the hydrogen atom ground state is $l=0$. Evidently, the radial coordinate $r$ is independent of the angular coordinates and it is a scalar in the 3 -dimensional space. The same is true for the contribution of the integration elements $\mathrm{d}^{3} r$. Hence, the angular momentum of the two sides of (2) is $l=0$. It follows that the angular momentum of bound fields is $l=0$. An analogous argument proves that the parity of bound fields is even.

Wigner's analysis of the inhomogeneous Lorentz group (see [8] [9] [10]) proves that a massive quantum particle is characterized by mass and spin whereas a massless particle is characterized by its helicity. Furthermore, if the quantum state of a particle is determined by a parity conserving interaction (like strong and electromagnetic interactions) then parity is a good quantum number and each particle has a well-defined parity.

These arguments prove that bound fields and radiation fields are different physical objects, because they have a different intrinsic angular momentum and a different parity. For these reasons, the following construction of the electromagnetic energy-momentum tensor treats separately radiation fields and bound fields. As explained above, this approach has a solid experimental basis.

\section{The Energy-Momentum Tensor of Radiation Fields}

Let us follow a standard construction of the energy-momentum tensor of electromagnetic fields (see [1], pp. 86, 87). The analysis begins with the electromagnetic fields Lagrangian density

$$
\mathcal{L}_{E M}=-\frac{1}{16 \pi} F^{\mu v} F_{\mu v}
$$


and applies the standard procedure for the construction of the energymomentum tensor. Here the electromagnetic 4-potential $A_{v}$ is used and one obtains

$$
T_{v}^{\mu}=\frac{\partial A_{\lambda}}{\partial x^{v}} \frac{\partial \mathcal{L}_{E M}}{\partial\left(\frac{\partial A_{\lambda}}{\partial x^{\mu}}\right)}-\delta_{v}^{\mu} \mathcal{L}_{E M}
$$

It is proved in [1] (p. 86) that a substitution of (3) into (4) yields the following form of the contravariant energy-momentum tensor

$$
T^{\mu v}=-\frac{1}{4 \pi} \frac{\partial A^{\lambda}}{\partial x_{\mu}} F_{\lambda}^{v}+\frac{1}{16 \pi} g^{\mu v} F^{\alpha \beta} F_{\alpha \beta} .
$$

The first term of the contravariant tensor (5) does not take a symmetric form whereas physical principles prove that "the energy-momentum tensor must be symmetric" (see [1], p. 84). This issue shows that the analysis described above apparently does not yield the required expression for the energy-momentum tensor. In particular, it is proved that the tensor (5) can be cast into a symmetric form if the following term

$$
\frac{1}{4 \pi} \frac{\partial A^{\mu}}{\partial x^{\lambda}} F^{v \lambda}=\frac{1}{4 \pi} \frac{\partial}{\partial x^{\lambda}}\left(A^{\mu} F^{v \lambda}\right)
$$

is added to it (see [1], near the bottom of p. 86 or [2], near the bottom of p. 604). The added term (6) is a 4-gradient and it does not alter the total energymomentum which is obtained from the spatial integrals of the appropriate entries of the energy-momentum tensor. This result shows the main problem which is discussed in this work: In the case of electromagnetic fields the variational principle does not yield the correct energy-momentum tensor and the quantity obtained is corrected by an auxiliary mathematical trick.

However, it is explained in the previous section that radiation fields and bound fields should be treated separately. Therefore, let us examine the form of the tensors (5) and (6) in the case of radiation fields. Here one can define axes so that the radiation looks locally as a plane wave which propagates in the $\mathrm{z}$-direction and is linearly polarized in the x-direction (see [1], pp. 118-120 and p. 184, after eq. (66.2)). The 4-potential of this wave is discussed in the literature. In appropriate units the physically meaningful mathematically real part of the 4 -potential of this plane wave is

$$
A_{\mu}=f(z-t)(0,1,0,0)
$$

(Note that in the units used herein $c=1$ and the argument of $F$ is $(z-t)$.)

The standard form of the electromagnetic field tensor is (see [1], p. 65)

$$
F^{\mu \nu}=g^{\mu \alpha} g^{\nu \beta}\left(A_{\beta, \alpha}-A_{\alpha, \beta}\right)=\left(\begin{array}{cccc}
0 & -E_{x} & -E_{y} & -E_{z} \\
E_{x} & 0 & -B_{z} & B_{y} \\
E_{y} & B_{z} & 0 & -B_{x} \\
E_{z} & -B_{y} & B_{x} & 0
\end{array}\right) .
$$


Let us calculate the correction term (6) in the case of the linearly polarized fields discussed herein (7). For these fields one finds that in the units where $c=1$, $|\boldsymbol{E}|=|\boldsymbol{B}|$ and only the components $E_{x}$ and $B_{y}$ do not vanish. Therefore, the tensor of the radiation fields discussed herein is

$$
F^{\mu \lambda}=f^{\prime}(z-t)\left(\begin{array}{cccc}
0 & -1 & 0 & 0 \\
1 & 0 & 0 & 1 \\
0 & 0 & 0 & 0 \\
0 & -1 & 0 & 0
\end{array}\right),
$$

where $f^{\prime}$ denotes the derivative of $f$. The 4-potential (7) depends only on the coordinates $z, t$. Hence, a direct calculation of the partial derivatives of the 4 -potential of (6) is

$$
\frac{\partial A^{\mu}}{\partial x^{\lambda}}=f^{\prime}(z-t)\left(\begin{array}{cccc}
0 & 0 & 0 & 0 \\
-1 & 0 & 0 & 1 \\
0 & 0 & 0 & 0 \\
0 & 0 & 0 & 0
\end{array}\right)
$$

A substitution of (9) and (10) into the correction term (6) proves that it is a null quantity. It follows that in the case of radiation fields, the energymomentum tensor (5) which is directly obtained from the variational principle is already symmetric. Its form can be written in the well known standard expression (see [1], p. 87)

$$
T^{\mu v}=\frac{1}{4 \pi}\left(-F^{\mu \lambda} F_{\lambda}^{v}+\frac{1}{4} g^{\mu v} F^{\alpha \beta} F_{\alpha \beta}\right) .
$$

The following example illustrates the significance of the symmetric form (11) of the energy-momentum tensor. The $T^{0 i}$ and the $T^{i 0}$ entries of (11) denote energy current and momentum density of the fields, respectively. In the symmetric tensor (11) they take the same form of the well-known Poynting vector (see [2], pp. 236-240)

$$
\boldsymbol{S}=\frac{1}{4 \pi} \boldsymbol{E} \times \boldsymbol{B}
$$

This example emphasizes the need for a symmetric energy-momentum tensor of electromagnetic fields.

\section{The Energy-Momentum Tensor of Bound Fields}

The construction of the energy-momentum tensor of bound fields is done separately for the case of charge-free space-time points and for space-time points that contain charge. At charge-free space-time points the charge's 4 -current $j^{\mu}=0$. Therefore, bound fields and radiation fields satisfy the same homogeneous Maxwell equations (see [1], pp. 71, 79)

$$
F_{, v}^{\mu v}=0 ; \quad F_{, v}^{* \mu v}=0 .
$$

Here the dual tensor of the second equation is $F^{* \mu \nu}=\epsilon^{\mu v \alpha \beta} F_{\alpha \beta}$ and $\epsilon^{\mu v \alpha \beta}$ is the completely antisymmetric unit tensor of the fourth rank. 
Since at charge-free space-time points radiation fields and bound fields satisfy the same equations of motion, one concluded that these fields have the same energy-momentum tensor (11). In order to complete the construction of the bound fields energy-momentum tensor it is required to show that (11) is suitable for space-time points where charge does not vanish. Here it is proved (see [1], pp. 88, 89) that the sum of the fields energy-momentum tensor $T_{F}^{\mu v}$ and that of charged matter $T_{M}^{\mu v}$ conserve energy-momentum

$$
\frac{\partial}{\partial x^{v}}\left(T_{F}^{\mu v}+T_{M}^{\mu v}\right)=0
$$

Therefore, the energy-momentum tensor (11) is a good expression for radiation fields and for bound fields as well.

As shown above, the energy-momentum tensor of bound fields is derived not from the Lagrangian density. Indeed, in a charge-free space where $j^{\mu}=0$, the derivation relies on the already known energy-momentum tensor of radiation fields and on the fact that the two kinds of fields satisfy the same equations of motion. If $j^{\mu} \neq 0$, the proof relies on an explicit calculation.

\section{Discussion}

A fundamental principle of physics says that a mathematical analysis of a given well established physical theory yields correct results. Therefore, the need for the correction term (6) which is added to the energy-momentum tensor (5) is inconsistent with this principle. This state of affairs proves the existence of a problem that should be settled. This dilemma is described in the quotation taken from [3] which is presented in the second paragraph of the Introduction section of this work. The novelty of this work is the proof that demonstrates that as a matter of fact the original tensor (5) is already symmetric provided the analysis is restricted to radiation fields. It is also proved above that the distinction between bound fields and radiation fields has a solid experimental basis. Therefore, the analysis must treat radiation fields and bound fields separately.

The following argument explains this conclusion. The analysis examines the hydrogen atom interaction with radiation fields and with bound fields and proves that in the quantum domain the spin-parity of radiation fields is $j^{\pi}=1^{-}$ whereas bound fields have $j^{\pi}=0^{+}$. Hence, in the quantum domain these fields should be treated separately. However, the Bohr correspondence principle shows that classical physics is the classical limit of quantum mechanics (see [5], pp. 15, $25-27,137,138)$. This important relationship between classical physics and quantum mechanics can be found in many textbooks. For example, "classical mechanics is contained in quantum mechanics as a limiting form" (see [6], p. 3). In particular, the electromagnetic fields term of the Lagrangian density (3) is used in the classical theory (see [1], p. 75) and in quantum theory (see [11], p. 70). Hence, the validity of the Bohr correspondence principle proves that the separation between radiation fields and bound fields must also take place in classical physics. 
Textbooks explain the drawbacks of the energy-momentum tensor (5) which is obtained from the electromagnetic Lagrangian density (see [1], pp. 82-86 or [2], p. 604). Let us examine the scientific meaning of the standard correction of the energy-momentum tensor which casts it into the symmetric form (6). The justification for this correction is that it is a 4-gradient which does not alter the integrals which yield the overall energy-momentum (see e.g. [1], pp. 83, 84). The following argument proves that this is just an arbitrary correction trick that has no profound basis.

Physics says that a mathematical analysis which is based on fundamental principles yields meaningful results. If adding a 4-gradient to a given energymomentum tensor is a legitimate procedure then instead of adding the term (6), one may multiply it by a factor $a \neq 1$ and add the new term. Evidently, the addition of the new term yields an unacceptable result.

In the scientific literature, the incorrect form of the energy-momentum tensor (5), which is directly obtained from the Lagrangian density, is called the canonical energy-momentum tensor (see [2], p. 601). The distinction between the canonical energy-momentum tensor and the physically acceptable symmetric energy-momentum tensor is still used in these days [12]. This is an indication of the novelty of the present work which proves that in the important case of radiation fields, the canonical energy-momentum tensor is already the physically acceptable symmetric tensor.

\section{Summary}

The problem of the apparently non-symmetric energy momentum tensor of electromagnetic fields which is derived from the variational principle is explained. The analysis relied on experimentally confirmed differences between radiation fields and bound fields. It is proved that in the case of radiation fields, a symmetric energy-momentum tensor is directly derived from the variational principle. Therefore, there is no need to fix this tensor by adding to it a correction term. It is also proved that the energy-momentum tensor of bound fields can be constructed from a mathematical analysis that does not directly depend on the variational principle. This work shows an example where the distinction between radiation fields and bound fields yields meaningful results. This distinction may be used in an analysis of other electromagnetic problems.

\section{References}

[1] Landau, L.D. and Lifshitz, M.E. (2005) The Classical Theory of Fields. Elsevier Amsterdam.

[2] Jackson, J.D. (1975) Classical Electrodynamics. John Wiley, New York.

[3] Munoz, G. (1996) Lagrangian Field Theories and Energy-Momentum Tensors. American Journal of Physics, 64, 1153-1157. https://doi.org/10.1119/1.18336

[4] Comay, E. (2015) Interrelations between Mathematics and Experiment in the Present Structure of Quantum Electrodynamics. Open Access Library Journal, 2, e2211. http://file.scirp.org/pdf/OALibJ_2016072610462207.pdf 
[5] Schiff, L.I. (1955) Quantum Mechanics. McGraw-Hill, New York.

[6] Merzbacher, E. (1970) Quantum Mechanics. John Wiley, New York.

[7] Patrignani C., et al. (Particle Data Group) (2016) The Review of Particle Physics. Chinese Physics C, 40, 100001. https://doi.org/10.1088/1674-1137/40/10/100001

[8] Wigner, E. (1939) On Unitary Representations of the Inhomogeneous Lorentz Group. Annals of Mathematics, 40, 149-204. https://doi.org/10.2307/1968551

[9] Schweber, S.S. (1964) An Introduction to Relativistic Quantum Field Theory. Harper \& Row, New York, 44-53.

[10] Sternberg, S. (1994) Group Theory and Physics. Cambridge University Press, Cambridge, 143-150.

[11] Bjorken, J.D. and Drell, S.D. (1965) Relativistic Quantum Fields. McGraw-Hill, New York.

[12] Wikipedia (2017) Stress-Energy Tensor. https://en.wikipedia.org/wiki/Stress\%E2\%80\%93energy_tensor\#Canonical_stress\% E2\%80\%93energy_tensor 